\title{
The Design Method of Axial Flow Runners Focusing on Axial Flow Velocity Uniformization and Its Application to an Ultra-Small Axial Flow Hydraulic Turbine
}

\author{
Yasuyuki Nishi, ${ }^{1}$ Yutaka Kobayashi, ${ }^{2}$ Terumi Inagaki, ${ }^{1}$ and Norio Kikuchi ${ }^{3}$ \\ ${ }^{1}$ Department of Mechanical Engineering, Ibaraki University, 4-12-1 Nakanarusawa-cho, Hitachi-shi, Ibaraki 316-8511, Japan \\ ${ }^{2}$ Graduate School of Science and Engineering, Ibaraki University, 4-12-1 Nakanarusawa-cho, Hitachi-shi, Ibaraki 316-8511, Japan \\ ${ }^{3}$ Ibasei, Ltd., 4-7-10 Kamine-cho, Hitachi-shi, Ibaraki 317-0064, Japan \\ Correspondence should be addressed to Yasuyuki Nishi; yasuyuki.nishi.fe@vc.ibaraki.ac.jp
}

Received 28 June 2016; Revised 21 October 2016; Accepted 3 November 2016

Academic Editor: Jechin Han

Copyright ( 2016 Yasuyuki Nishi et al. This is an open access article distributed under the Creative Commons Attribution License, which permits unrestricted use, distribution, and reproduction in any medium, provided the original work is properly cited.

\begin{abstract}
We proposed a portable and ultra-small axial flow hydraulic turbine that can generate electric power comparatively easily using the low head of open channels such as existing pipe conduits or small rivers. In addition, we proposed a simple design method for axial flow runners in combination with the conventional one-dimensional design method and the design method of axial flow velocity uniformization, with the support of three-dimensional flow analysis. Applying our design method to the runner of an ultra-small axial flow hydraulic turbine, the performance and internal flow of the designed runner were investigated using CFD analysis and experiment (performance test and PIV measurement). As a result, the runners designed with our design method were significantly improved in turbine efficiency compared to the original runner. Specifically, in the experiment, a new design of the runner achieved a turbine efficiency of 0.768 . This reason was that the axial component of absolute velocity of the new design of the runner was relatively uniform at the runner outlet in comparison with that of the original runner, and as a result, the negative rotational flow was improved. Thus, the validity of our design method has been verified.
\end{abstract}

\section{Introduction}

Conventionally, hydropower generation has been used in large-scale facilities to generate electric power efficiently by utilizing high or medium hydraulic head. However, the number of locations where enough head is available is decreasing. In addition, installation of large-scale facilities requires largescale civil engineering projects, which have a significant impact on the environment. Therefore, it is thought that small hydraulic turbines show promise in generating power in situations of low available head; such turbines would use water in open channels such as existing pipe conduits or small rivers [1-4]. It is also expected that because axial flow hydraulic turbines are particularly suited for low head applications, they will be widely implemented if they are decreased in size and made portable [5-8]. However, small axial flow hydraulic turbines have extremely low Reynolds numbers (approximately $1 \times 10^{5}$ ); thus, very few airfoils are applicable. Furthermore, design methods are not well established.

Incidentally, in hydraulic turbines, the design of the Francis hydraulic turbine has been optimized in recent years using a design of experiments and an optimization algorithm [9-11]. However, a large amount of sample data needs to be acquired and obtaining the most feasible solution is time consuming; thus, the computational load increases. Therefore, it is important to establish a simple design method for designing reliable and highly efficient axial flow runners from the null state without sample data.

In light of this background, in this study, we propose a portable and ultra-small axial flow hydraulic turbine that can generate electric power using the low head of open channels such as existing pipe conduits or small rivers. The runners and guide vanes of this hydraulic turbine are designed using a conventional one-dimensional design method [12, 13], and their performance and internal flows are investigated 
by numerical analysis. In addition, we propose a simple design method for axial flow runners in combination with the conventional one-dimensional design method and the design method of axial flow velocity uniformization, with the support of three-dimensional flow analysis. This design method can decide the unique form of the runner on the basis of the quantitative data of the velocity distributions at the runner inlet and outlet using three-dimensional flow analysis. Therefore, we believe that our method can provide reliable and highly efficient performance for a runner in comparison with the method wherein designers empirically repeat the changes of shapes by examining the analytical results from complicated three-dimensional internal flows. Applying our design method to the runner of an ultra-small axial flow hydraulic turbine, the performance and internal flow of the designed runner are investigated using numerical analysis. In addition, as verification, we conducted an experiment using an actual device and verified the validity of this design method.

\section{Simple Design Method for Axial Flow Runners}

In this study, we propose a simple design method for axial flow runners, focusing on axial flow velocity uniformization. This design method is a combination of the conventional onedimensional design method $[12,13]$ and the design method of axial flow velocity uniformization, with the support of a three-dimensional flow analysis. The design flowchart is shown in Figure 1. First, a runner is designed on the basis of the conventional one-dimensional design method. Velocity distributions at the inlet and outlet of the runner are examined on the basis of the three-dimensional flow analysis. Next, the axial flow velocity uniformization is performed, and the blade angle and chord length of the runner are modified. The performance of the runner is verified using three-dimensional flow analysis. If it does not achieve the target performance, the axial flow velocity uniformization is performed again. Through iterations of this procedure, the design process is complete when the performance achieves its target. We have assumed that this design method is effective in cases wherein factors such as a low Reynolds number or characteristic data of the airfoil are unclear and the assumed flow by the one-dimensional design method strongly deviates from the actual three-dimensional flow. The following are the details of the conventional design method $[12,13]$ and the design method of axial flow velocity uniformization.

2.1. One-Dimensional Design Method. The specific speed $n_{s}$ is calculated using the following design parameters: effective head $H$, turbine output $L$, and rotational speed $n$.

$$
n_{s}=\frac{n(L / 1000)^{1 / 2}}{H^{5 / 4}} .
$$

Using the specific speed, $n_{s}$, obtained from (1) and a design diagram [12], the circumferential velocity coefficient $k_{u}$, hub ratio $v$, and the axial velocity coefficient $k_{a}$ are calculated. In addition, the circumferential velocity $u_{t}$ of a tip,

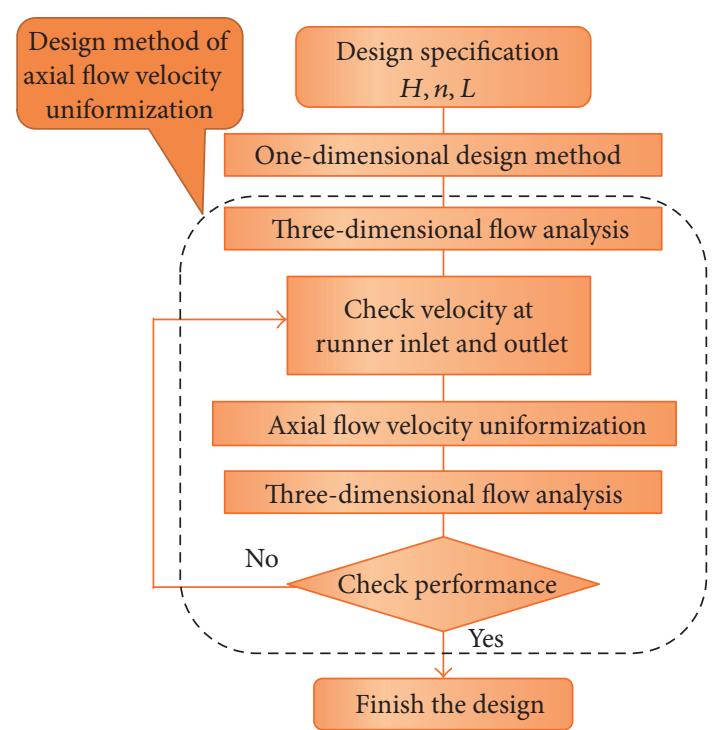

Figure 1: Design flow.

the outer diameter $D_{t}$ of a runner, and the hub diameter $D_{h}$ are obtained with the following formulae:

$$
\begin{aligned}
u_{t} & =k_{u} \sqrt{2 g H}, \\
D_{t} & =\frac{60 u_{t}}{\pi n}, \\
v & =\frac{D_{h}}{D_{t}} .
\end{aligned}
$$

Furthermore, the axial component $v_{a}$ of the absolute velocity and the flow rate $Q$ are obtained with the following formulae:

$$
\begin{aligned}
& v_{a}=k_{a} \sqrt{2 g H}, \\
& Q=\frac{\pi\left(1-v^{2}\right) D_{t}^{2} v_{a}}{4} .
\end{aligned}
$$

Next, dividing the blade into several parts from hub to tip, vortex design is determined. Referencing specific speed $n_{s}$, the number of blades $z$ is determined, and then the pitch $t$ at each radial point $\varphi$ is calculated by the following expression:

$$
t=\frac{\pi D}{z} \text {. }
$$

The velocity triangle of this runner is illustrated in Figure 2 [13]. At each radial point $\varphi$, the airfoil and attack angle $\delta$ are selected. By estimating hydraulic efficiency $\eta_{h}$, chord length $l$ and mean relative flow angle $\bar{\beta}$ between the runner inlet and outlet are calculated using the following expression [13]:

$$
\begin{aligned}
\bar{w} & =\frac{v_{a}}{\sin \bar{\beta}}, \\
\eta_{h} H & =\frac{1}{2 g} \frac{l}{t} C_{L} u \bar{w}(1-\varepsilon \cot \bar{\beta}) .
\end{aligned}
$$




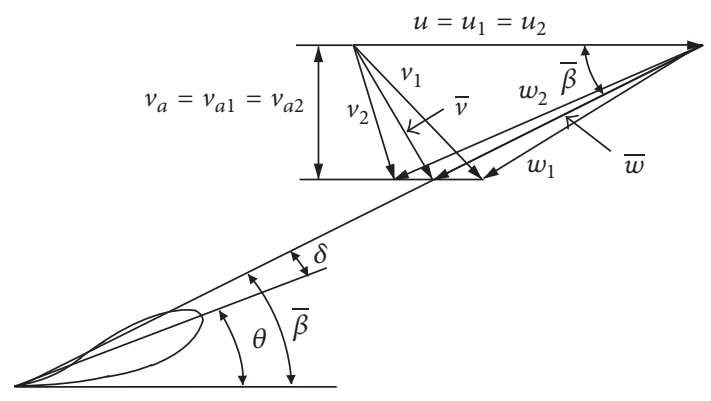

FIGURE 2: Velocity triangle.

In this study, the blade is divided into four parts from hub to tip, and the calculation is performed at five radial points. This vortex design type is a free vortex. At each radial point $\varphi$, the airfoil selects MEL031 [14] in which characteristic data exist for low Reynolds numbers. The attack angle is $\delta=2^{\circ}$. However, considering that the Reynolds number based on chord length and relative velocity is as low as approximately $1 \times 10^{5}$, hydraulic efficiency is estimated to be $\eta_{h}=0.7(\eta=$ $0.659)$.

In addition, the blade angle $\theta$ is obtained from the following formula:

$$
\theta=\bar{\beta}-\delta
$$

Finally, taking into account the leakage loss, the blade tip clearance is determined.

The one-dimensional design method for the guide vane is also shown below. The outer diameter of the guide vane is the size of the outer diameter of the runner plus the blade tip clearance, and the hub diameter is the same as that of the runner. Dividing the blade into several parts from hub to tip, vortex design is determined. Referencing specific speed $n_{s}$, the number of blades $z_{g}$ of the guide vane is determined, and then the pitch $t_{g}$ of the guide vane at each radial point $\varphi_{g}$ is calculated by the following expression:

$$
t_{g}=\frac{\pi D_{g}}{z_{g}} .
$$

At each radial point $\varphi_{g}$, the airfoil and attack angle $\delta_{g}$ of the guide vane are selected. It is assumed that prerotation at a guide vane inlet is zero $\left(v_{u 3}=0\right)$ and that $v_{u 4}=v_{u 1}$ at a guide vane outlet. Mean absolute flow angle $\overline{\alpha_{g}}$ between the guide vane inlet and outlet and blade angle $\theta_{g}$ are calculated using the following expression [15]:

$$
\begin{aligned}
\tan \overline{\alpha_{g}} & =\frac{\overline{v_{a g}}}{\left(\left(v_{u 3}+v_{u 4}\right) / 2\right)}, \\
\theta_{g} & =\overline{\alpha_{g}}-\delta_{g},
\end{aligned}
$$

where $\overline{v_{a g}}$ is the axial component of the mean absolute velocity between the guide vane inlet and outlet.
In addition, the chord length $l_{g}$ of the guide vane is obtained from the following formula [15]:

$$
C_{L g} \frac{l_{g}}{t_{g}}=\frac{2 v_{u 4}}{\overline{v_{a g}}} \frac{\cos \varepsilon_{g} \sin ^{2} \overline{\alpha_{g}}}{\sin \left(\overline{\alpha_{g}}+\varepsilon_{g}\right)} .
$$

In this study, the blade of the guide vane is divided into four parts from hub to tip, and the calculation is performed at five radial points. This vortex design type is a free vortex. At each radial point $\varphi_{g}$, the airfoil selects MEL031 [14], and the attack angle is $\delta_{g}=8^{\circ}$.

2.2. Design Method of Axial Flow Velocity Uniformization. By performing a three-dimensional flow analysis for the runner designed with the one-dimensional design method shown in Section 2.1, the distributions of the axial component of the absolute velocity at the runner inlet and outlet are calculated. Using these velocity distributions, axial flow velocity uniformization is performed. Specifically, the axial components $\overline{v_{a c}}$ of the mean absolute velocities between the runner inlet and outlet are calculated on the basis of the values analyzed at each radial point $\varphi$. Then after the axial flow velocity uniformization is performed, the axial components $\overline{v_{a}^{\prime}}$ of the mean absolute velocities between the runner inlet and outlet are obtained from the following expression:

$$
\overline{v_{a}^{\prime}}=\overline{v_{a d}}+\left(\overline{v_{a d}}-\overline{v_{a c}}\right)
$$

Here, $\overline{v_{a d}}$ is the axial component of the mean absolute velocity between the runner inlet and outlet based on the design values using the one-dimensional design method.

Accordingly, after performing the axial flow velocity uniformization, the chord length $l^{\prime}$ and the blade angle $\theta^{\prime}$ are obtained from formulae (11).

$$
\begin{aligned}
& \overline{\beta^{\prime}}=\tan ^{-1}\left(\frac{\overline{v_{a}^{\prime}}}{\overline{w_{d}} \cos \overline{\beta_{d}}}\right), \\
& l^{\prime}=\frac{2 g H \eta_{h} t}{C_{L} u \overline{w_{d}}\left(1-\varepsilon \cot \overline{\beta^{\prime}}\right)}, \\
& \theta^{\prime}=\overline{\beta^{\prime}}-\delta .
\end{aligned}
$$

Here, $\overline{w_{d}}$ is the mean relative velocity between the runner inlet and outlet based on the design values using the onedimensional design method.

In addition, $\overline{\beta_{d}}$ is the mean relative flow angle between the runner inlet and outlet based on the design values using the one-dimensional design method.

As shown above, the chord length $l^{\prime}$ and the blade angle $\theta^{\prime}$ at each radial point $\varphi$ are modified, and then the three-dimensional flow analysis of the modified runner is performed. The verification of performance and the axial flow velocity uniformization are iterated until the target performance is obtained. In this study, the target performance for the turbine efficiency is $\eta=0.75$. 


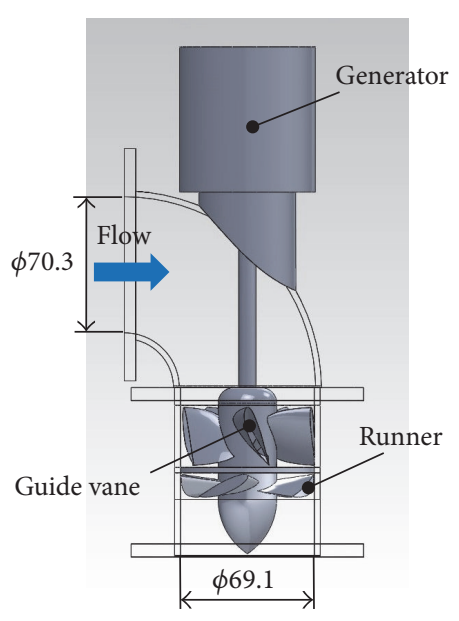

FIGURE 3: Ultra-small axial flow hydraulic turbine.

\section{Test Hydraulic Turbine}

An overview of the ultra-small axial flow hydraulic turbine proposed in this study is shown in Figure 3. This hydraulic turbine is easily portable. The targets are set as follows: effective head $H=1.5 \mathrm{~m}$, turbine output $L=100 \mathrm{~W}$ at a rotational speed of $n=2460 \mathrm{~min}^{-1}$, and a turbine efficiency of $\eta=0.75$, so that it is possible to obtain a practical turbine output when the turbine is implemented at low head values. The specific speed $n_{s}$ shown in formula (1) is $469\left[\mathrm{~min}^{-1}, \mathrm{~kW}\right.$, $\mathrm{m}]$. Considering that the Reynolds number based on chord length and relative velocity is as low as approximately $1 \times$ $10^{5}$, the design values in the one-dimensional design method are set as follows: effective head $H=1.5 \mathrm{~m}$, turbine output $L$ $=75.5 \mathrm{~W}$ at rotational speed $n=2460 \mathrm{~min}^{-1}$, and turbine efficiency $\eta=0.659$.

An overview of the sample runners is shown in Figures 4(a)-4(d), and the dimensions are shown in Table 1. Here the original runner is designed using only the conventional onedimensional design method shown in Section 2.1. The outer diameter of the runner is $D_{t}=68.1 \mathrm{~mm}$, the hub diameter is $D_{h}=30.2 \mathrm{~mm}$, the number of blades is $z=4$, and the blade tip clearance is $0.5 \mathrm{~mm}$. In addition, MEL031 [11] is selected for an airfoil at any radial point, and the attack angle is $\delta=2^{\circ}$. The design flow rate is $Q=0.0078 \mathrm{~m}^{3} / \mathrm{s}$. Case 1 runner, Case 2 runner, and Case 3 runner were all designed using the design method proposed in this study and were created after the axial flow velocity uniformization was performed once, twice, and three times, respectively.

An overview of the sample guide vane is shown in Figure 5, and the dimensions are shown in Table 2. The guide vane is designed using the conventional one-dimensional design method shown in Section 2.1. The outer diameter is $D_{t g}=69.1 \mathrm{~mm}$, the hub diameter is $D_{h g}=30.2 \mathrm{~mm}$, and the number of blades is $z_{g}=5$. In addition, MEL031 [14] is selected for an airfoil at any radial point, and the attack angle is $\delta_{g}=8^{\circ}$.
TABLE 1: Specifications of test runners.

\begin{tabular}{|c|c|c|c|c|}
\hline & & Hub & Mid & Tip \\
\hline \multirow{5}{*}{ Original } & Radius $r$ [mm] & 15.1 & 24.6 & 34.1 \\
\hline & Solidity $l / t$ & 1.04 & 0.72 & 0.57 \\
\hline & Blade angle $\theta\left[^{\circ}\right]$ & 16.9 & 21.0 & 24.7 \\
\hline & Airfoil & & MEL031 & \\
\hline & Blade number $z$ & & 4 & \\
\hline \multirow{5}{*}{ Case 1} & Radius $r$ [mm] & 15.1 & 24.6 & 34.1 \\
\hline & Solidity $l / t$ & 0.97 & 0.72 & 0.58 \\
\hline & Blade angle $\theta\left[^{\circ}\right]$ & 31.7 & 27.8 & 31.0 \\
\hline & Airfoil & & MEL031 & \\
\hline & Blade number $z$ & & 4 & \\
\hline \multirow{5}{*}{ Case 2} & Radius $r$ [mm] & 15.1 & 24.6 & 34.1 \\
\hline & Solidity $l / t$ & 0.95 & 0.72 & 0.59 \\
\hline & Blade angle $\theta\left[{ }^{\circ}\right]$ & 36.4 & 19.1 & 11.3 \\
\hline & Airfoil & & MEL031 & \\
\hline & Blade number $z$ & & 4 & \\
\hline \multirow{5}{*}{ Case 3} & Radius $r$ [mm] & 15.1 & 24.6 & 34.1 \\
\hline & Solidity $l / t$ & 0.95 & 0.73 & 0.60 \\
\hline & Blade angle $\theta\left[^{\circ}\right]$ & 38.0 & 17.0 & 7.6 \\
\hline & Airfoil & & MEL031 & \\
\hline & Blade number $z$ & & 4 & \\
\hline
\end{tabular}

TABLE 2: Specifications of guide vane.

\begin{tabular}{lccc}
\hline & Hub & Mid & Tip \\
\hline Radius $r_{g}[\mathrm{~mm}]$ & 15.1 & 24.8 & 34.6 \\
Solidity $l_{g} / t_{g}$ & 1.53 & 1.01 & 0.74 \\
Blade angle $\theta_{g}\left[^{\circ}\right]$ & 54.8 & 64.6 & 69.3 \\
Airfoil & & MEL031 & \\
Blade number $z_{g}$ & & 5 & \\
\hline
\end{tabular}

\section{Numerical Analysis Methods and Conditions}

The computational model is shown in Figure 6. As shown in Figure 3, a bent pipe was installed in the upper flow of the guide vane in the case of a real hydraulic turbine. However, to simplify the analytical model and remove the influence of the bent pipe, we used a computational model comprising only a direct pipe when we applied our design method. We believe that the tendency of the qualitative performance of this hydraulic turbine does not change depending on the presence of the bent pipe. We used the general-purpose thermal fluid analysis code ANSYS CFX15.0 for the numerical analyses and conducted three-dimensional steady flow analyses. The governing equations are the conservation of mass equation [16] and the conservation of momentum equation [16]. The SST (Shear Stress Transport) model [16] was adopted as the turbulence model. Water was used for the working fluid. For boundary conditions, the mass flow rate $7.793 \mathrm{~kg} / \mathrm{s}(Q=$ $0.0078 \mathrm{~m}^{3} / \mathrm{s}$ ) was applied to the inlet boundary, static pressure (gauge pressure) $0 \mathrm{~Pa}$ was applied to the outlet boundary, and arbitrary rotational speed was applied to the runner 


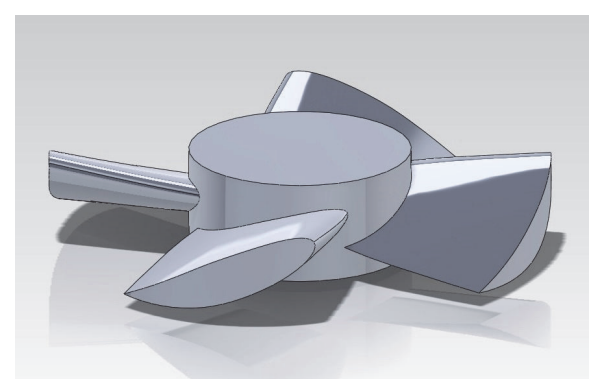

(a) Original

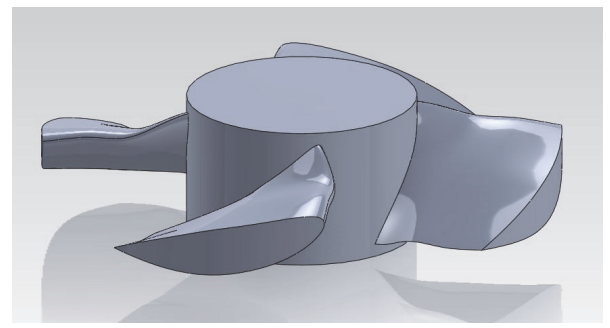

(c) Case 2

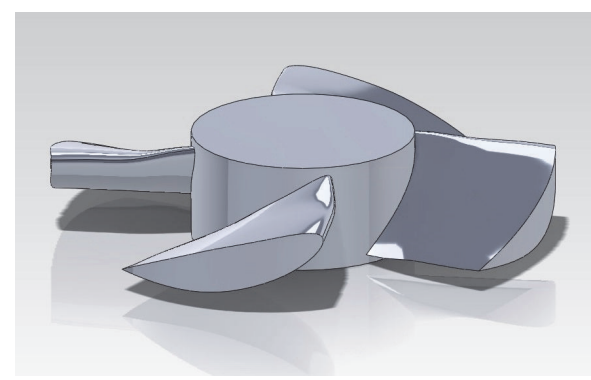

(b) Case 1

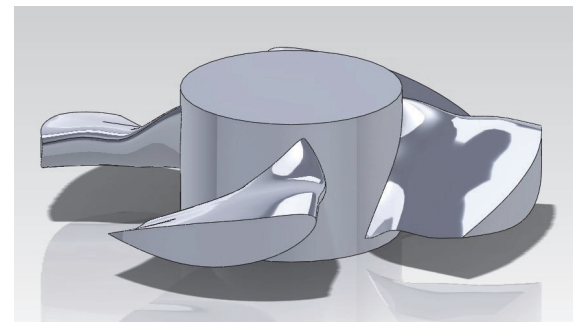

(d) Case 3

Figure 4: Test runners.

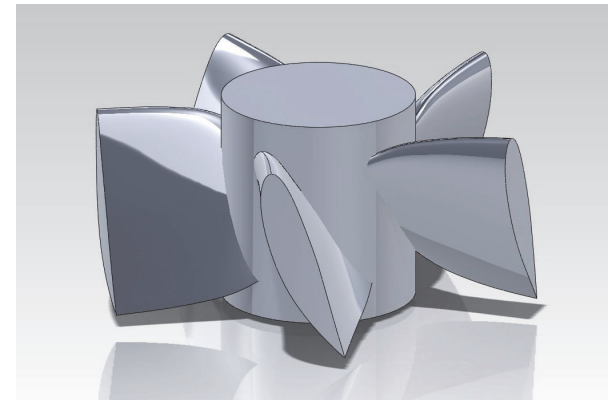

FIGURE 5: Guide vane.

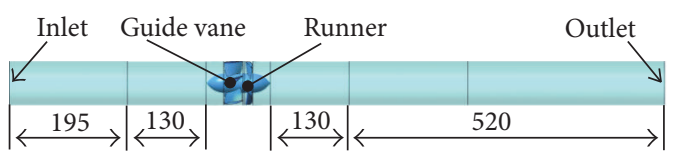

FIgUre 6: Computational domain.

region. Nonslip conditions were applied to all the walls. Moreover, the boundaries between the rotating and static systems were joined using the frozen rotor [17]. For example, the computational grids of the original runner and the guide vane are shown in Figures 7(a) and 7(b). The computational grid of each runner was a tetrahedron, and the face size of the blade surface was $0.4 \mathrm{~mm}$. There were five prism layers of the blade surface, and the size of the first layer was $0.03 \mathrm{~mm}$. The numbers of computational elements for original runner, Case 1 runner, Case 2 runner, and Case 3 runner, were approximately 2,070,000, 2,130,000, 2,600,000, and 2,810,000 elements, respectively. The computational grid of the guide vane was a tetrahedron, and the face size of the blade surface was $0.6 \mathrm{~mm}$. There were five prism layers of the blade surface, and the size of the first layer was $0.03 \mathrm{~mm}$. The number of computational elements for the guide vane was approximately $1,280,000$. The total numbers of computational elements for original runner, Case 1 runner, Case 2 runner, and Case 3 runner, were approximately $5,060,000,5,110,000,5,580,000$, and 5,800,000 elements, respectively. To study the grid dependence, we performed an analysis after increasing the computational elements for the original runner and guide vane to approximately $3,170,000$ and 1,990,000, respectively; then, we performed an analysis after increasing the computational elements to approximately $4,250,000$ and 2,550,000, respectively. Consequently, it was confirmed that the effect of the number of computational elements in the grids was relatively low even though the effective head and turbine output changed by approximately $+3.0 \%$ and $-0.9 \%$, respectively, in the former analysis and by approximately $+2.6 \%$ and $-1.2 \%$, respectively, in the latter analysis. Moreover, we performed an unsteady flow analysis for the original runner using the transient rotor-stator [17]. As a result, it was confirmed that the difference between the steady and unsteady flow analyses was relatively small even though the effective head and turbine output changed by approximately $+2.5 \%$ and $+3.3 \%$, respectively, compared with the results presented in the paper.

\section{Analysis Results and Discussion}

5.1. Comparison of Turbine Performance. The turbine performance of each runner obtained by numerical analysis is depicted in Figures $8(\mathrm{a})-8(\mathrm{c})$. In the figures, the design value of the turbine output $(75.5 \mathrm{~W})$ is also illustrated. For the original runner, the maximum value of turbine efficiency, $\eta$, is 0.662 at the design rotational speed $n=2460$ and is almost equal to the design value (0.659). However, the turbine output 


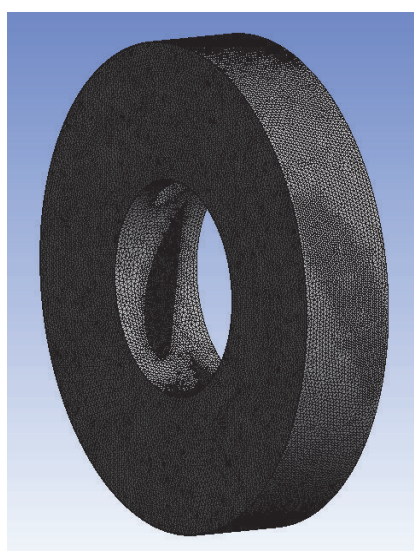

(a) Runner (original)

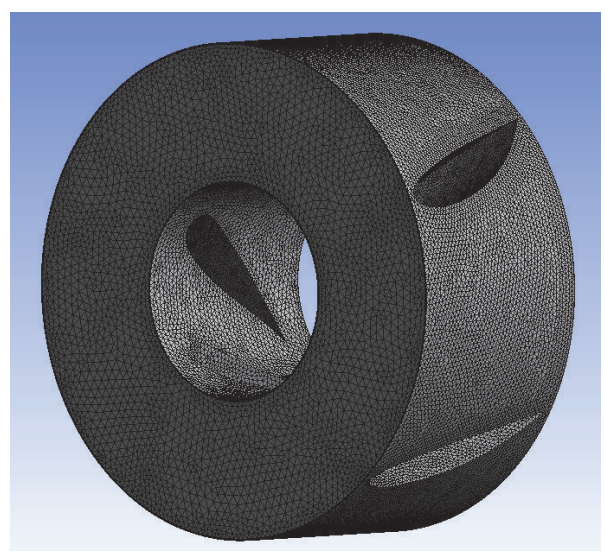

(b) Guide vane

FIGURE 7: Computational grids.

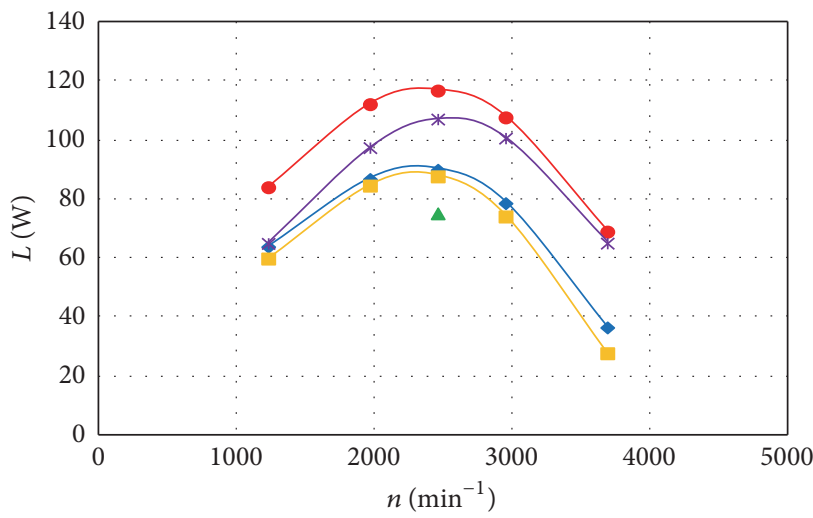

$\rightarrow$ Original

$\rightarrow$ Case 1

- Case 2

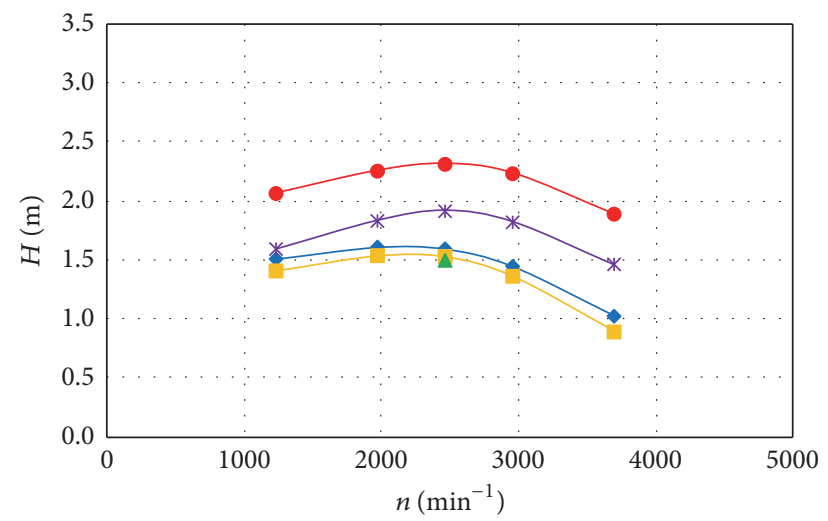

$\rightarrow$ Original

$\rightarrow$ Case 1

- Case 2

* Case 3

- Design value

(b) Effective head

(a) Turbine output

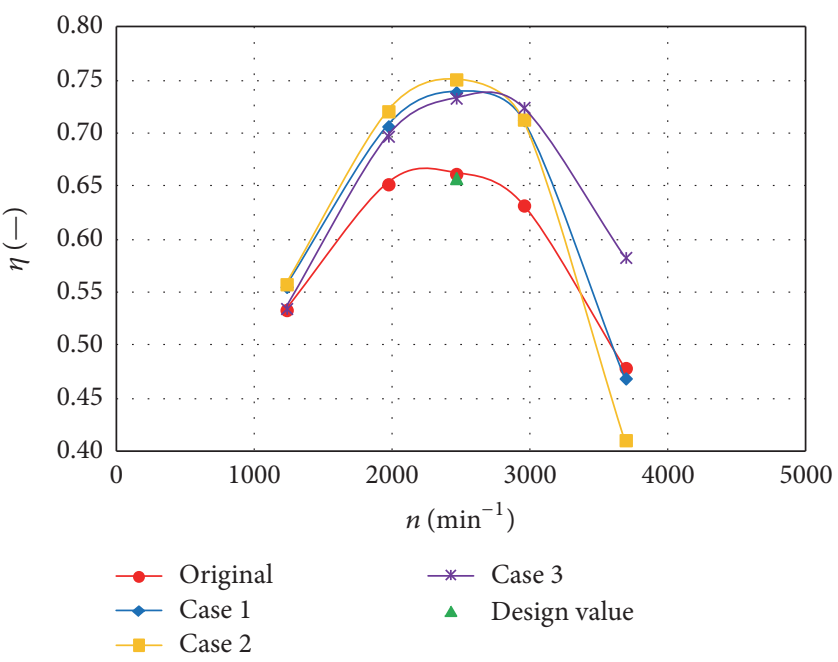

(c) Turbine efficiency

FIgURE 8: Turbine performances (Cal.). 


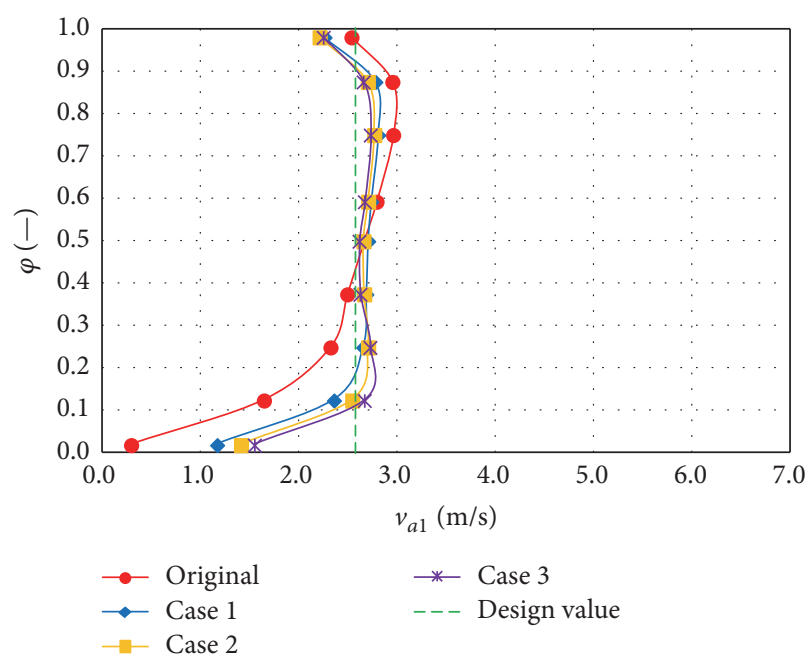

(a) Axial component

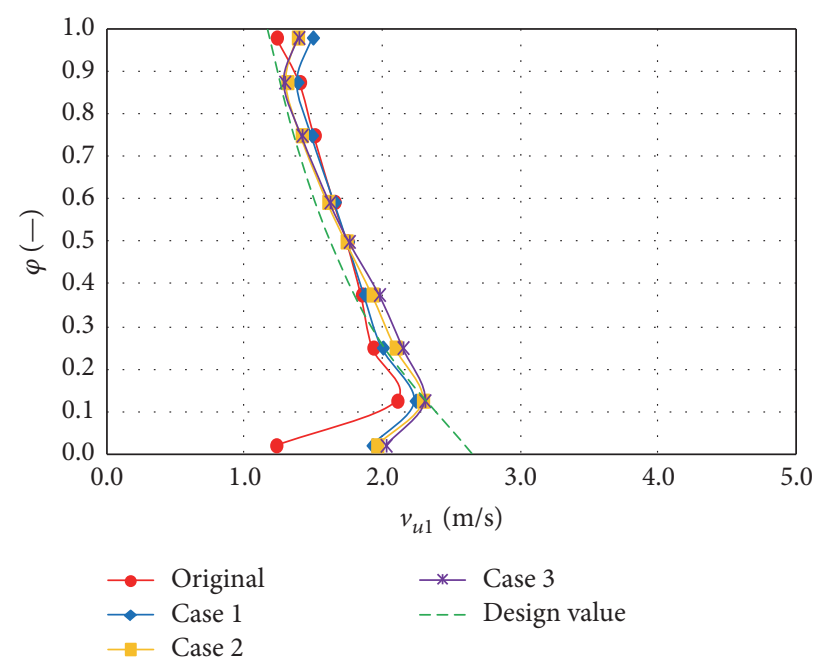

(b) Circumferential component

Figure 9: Absolute velocity at runner inlet $\left(n=2460 \mathrm{~min}^{-1}\right.$, Cal.).

$L$ and the effective head $H$ are significantly larger than their design values. By contrast, for Cases 1-3 runners designed using our design method, the turbine output, $L$, decreases in all the rotational speed regions more than that of the original runner. However, at the same time, the effective head, $H$, decreases. Therefore, the turbine efficiency, $\eta$, of Cases 1-3 runners, except at $n=3690$, have all significantly improved when compared to that of the original runner. It is noted that, in particular, Case 2 runner achieves a turbine efficiency of $\eta=0.752$ at $n=2460$, which surpasses the design value. However, $\eta$ value of Case 3 runner is slightly lower than that of Case 2 runner. This appears to be because the hydraulic efficiency $\eta_{h}$ that was assumed in the design of the axial flow velocity uniformization is different from actual values.

5.2. Comparison of Internal Flow. The axial component $v_{a 1}$ and the circumferential component $v_{u 1}$ of the absolute velocity at the runner inlet at each radial point $\varphi$ of each runner are illustrated in Figures 9(a) and 9(b). Here, $v_{a 1}$ and $v_{u 1}$ are the circumferential average values of the axial component and the circumferential component, respectively, at the $1 \mathrm{~mm}$ upper point of the stream from the blade. Rotational speed is $n=$ 2460 . The value $\varphi=0$ represents the hub, and $\varphi=1$ represents the tip. The value $v_{a 1}$ of each runner decreases significantly on the hub side. This phenomenon can be explained by the Reynolds number decreasing on the hub side, a boundary layer developing on the hub surface when flow passes through a guide vane, and other related effects. Therefore, $v_{a 1}$ is slightly larger than the design value from the mid-point to the tip-point, but it is close to the design value as a whole. In particular, Cases 1-3 runners, unlike the original runner, decrease nonuniformity and have a similar distribution of design values. The value $v_{u 1}$ is in relatively good agreement with the design value although, for each runner, the value decreases on the hub side. In particular, in Cases 1-3 runners, the diminution of $v_{u 1}$ near the hub is smaller compared to the original runner, and thus the distribution gets closer to that of the design. Based on the discussion in the preceding text, the design of the guide vane is appropriate to some extent.

The axial component $v_{a 2}$ and the circumferential component $v_{u 2}$ of the absolute velocity at the runner outlet at each radial point $\varphi$ of each runner are illustrated in Figures $10(\mathrm{a})$ and $10(\mathrm{~b})$. Here, $v_{a 2}$ and $v_{u 2}$ are the circumferential average values of the axial component and the circumferential component, respectively, at the $1 \mathrm{~mm}$ lower point of the stream from the blade. Rotational speed is $n=2460$. The value $\varphi=0$ represents the hub, and $\varphi=1$ represents the tip. In the case of the original runner, $v_{a 2}$ is smaller on the hub side and becomes larger toward the tip. In addition, $v_{u 2}$ has negative values except for the values on the tip side, thereby indicating a negative rotation, that is, in the direction opposite to the direction of flow into the runner. Causes of this might be that the Reynolds number of this runner is low, which becomes even lower for locations closer to the hub, or that an axial flow velocity at the runner inlet was not uniform previously or that the boundary layer develops on the hub surface, among other reasons. This negative rotation flow appears to increase the effective head and the turbine output. In contrast, Cases 1-3 runners designed with our design method have a value of $v_{a 2}$ that, in comparison to that of the original runner, is uniform and close to the design value; they also have a value of $v_{u 2}$ that is also close to the design value. Case 2 runner is particularly noteworthy. As shown in Figures 11(a) and 11(b), if the absolute velocity vectors are compared between the original runner and Case 2 runner, the reverse flow is created at the runner outlet of the original runner but is eliminated in Case 2 runner. Accordingly, it is apparent that the turbine efficiency of Case 2 runner is significantly improved compared to the original runner.

\section{Verification by Experiment}

6.1. Experimental Apparatus and Methods. To verify the high performance of the hydraulic turbine described in Section 5, 


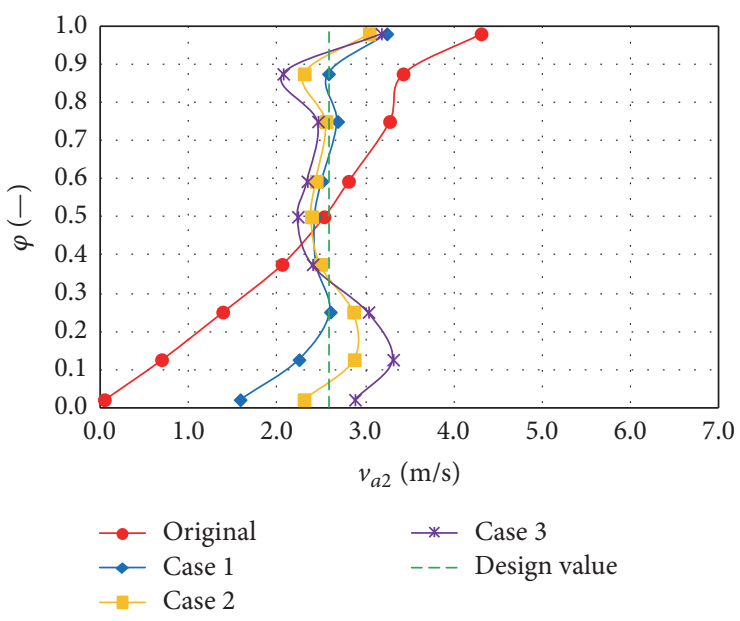

(a) Axial component

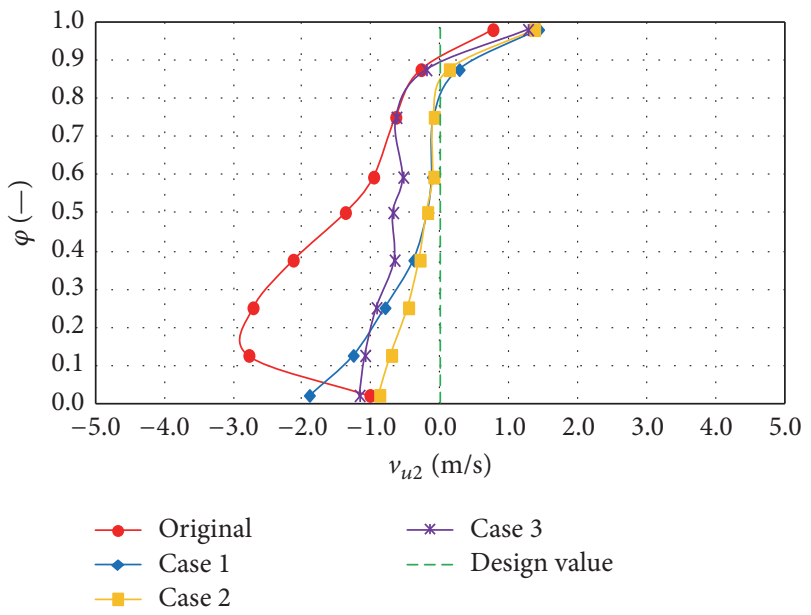

(b) Circumferential component

FIGURE 10: Absolute velocity at runner outlet $\left(n=2460 \mathrm{~min}^{-1}\right.$, Cal.).

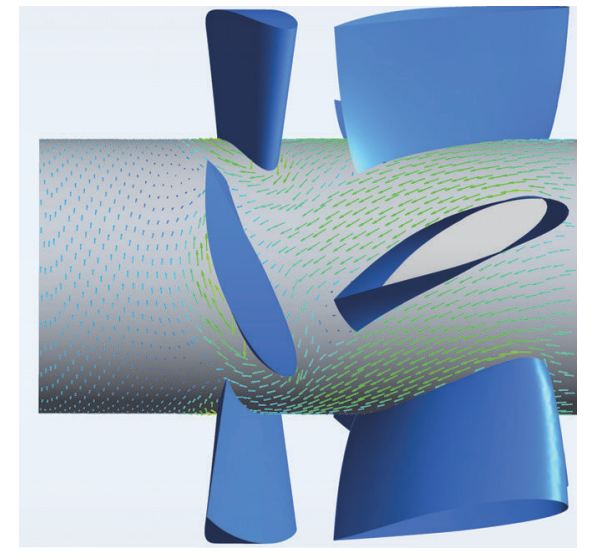

(a) Original

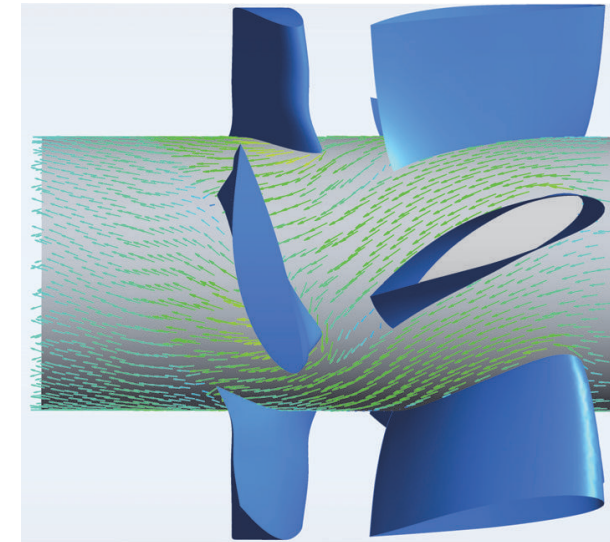

(b) Case 2

FIGURE 11: Absolute velocity vector $\left(\varphi=0.125, n=2460 \mathrm{~min}^{-1}\right.$, Cal. $)$.

an actual device was used for a verification test. An overview of the experimental apparatus is shown in Figure 12. Actual devices of the runners are shown in Figures 13(a) and 13(b), and an actual device of the guide vane is shown in Figure 14. Water was used for the working fluid, and the experiment was conducted with a constant flow rate $Q=0.0078 \mathrm{~m}^{3} / \mathrm{s}$. The flow rate was measured using an electromagnetic flow meter (TOSHIBA CORPORATION; GF630, Accuracy $\pm 0.5 \%$ of rate). The load of the hydraulic turbine was controlled using a motor and an inverter, and the rotational speed $n$ was set up arbitrarily. The rotational speed $n$ and torque $T$ were measured with a magnetoelectric-type rotation detector (Ono Sokki Co., Ltd.; MP-981, accuracy $\pm 0.02 \%$ of full scale) and a torque detector (Ono Sokki Co., Ltd.; SS-005, accuracy $\pm 0.2 \%$ of full scale), respectively. From these values, the turbine output $L$ was obtained.

$$
L=\frac{2 \pi n T}{60} .
$$

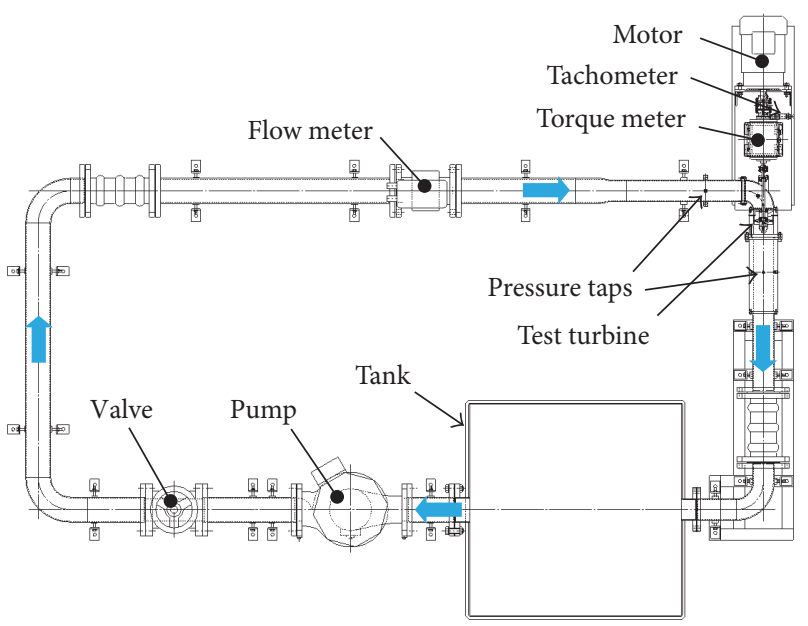

FIGURE 12: Experimental apparatus. 


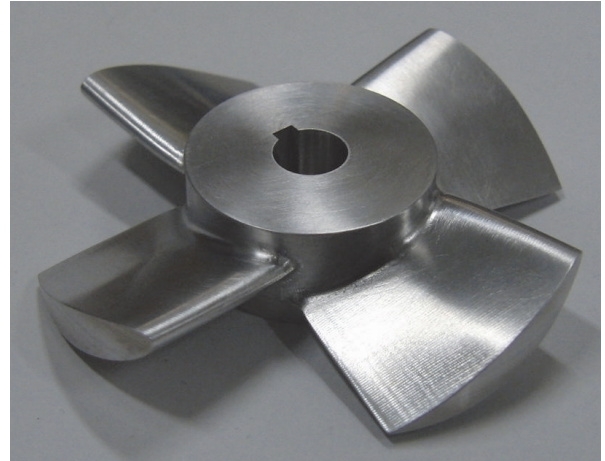

(a) Original

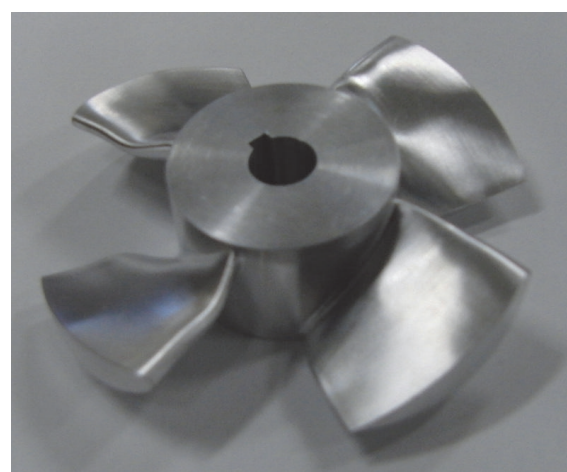

(b) Case 2

FIGURE 13: Actual device of the runner.

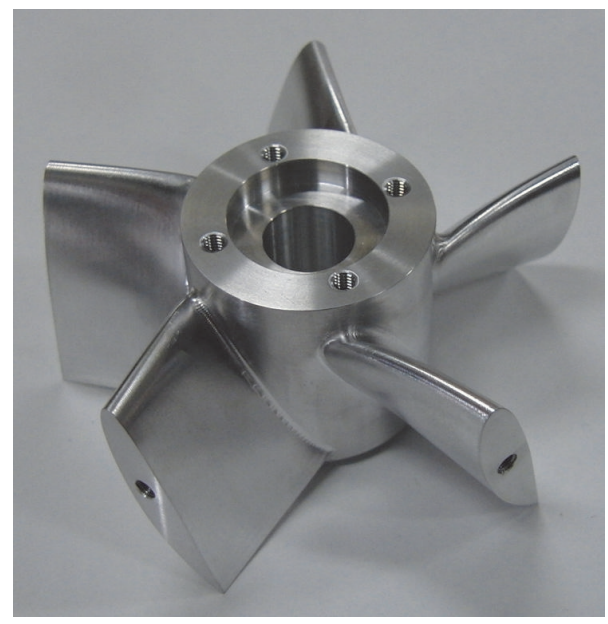

FIGURE 14: Actual device of the guide vane.

Note that the torque obtained was corrected by measuring the torque without a runner. The static pressures at the inlet and outlet of a hydraulic turbine were measured with a straingauge pressure transducer (Kyowa Electronic Instruments Co., Ltd.; PGMC-A-200KP-F, nonlinearity $\pm 1.5 \%$ rated output). The effective head, $H$, was calculated using the difference between the static pressures and the difference between the dynamic pressures obtained from the flow rate.

$$
H=\frac{P_{i}-P_{o}}{\rho g}+\frac{v_{i}^{2}-v_{o}^{2}}{2 g} .
$$

Here, $P_{i}$ and $P_{o}$ are the static pressure of the turbine inlet and outlet. In addition, $v_{i}$ and $v_{o}$ are the average flow velocity of the turbine inlet and outlet.

Furthermore, the turbine efficiency $\eta$ was obtained with the following formula:

$$
\eta=\frac{L}{\rho g Q H}
$$

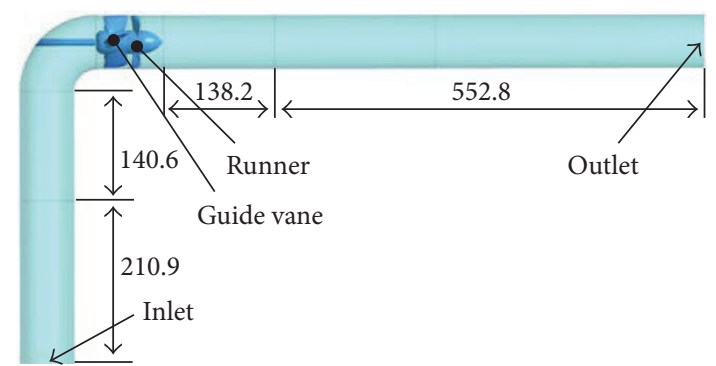

FIGURE 15: Computational domain.

For the measurement errors for the original runner at $n=$ 2460, the total errors of the turbine output $L$, effective head $H$, and turbine efficiency $\eta$ were approximately $\pm 4.7 \%( \pm 0.12 \mathrm{~m})$, $\pm 2.7 \%( \pm 3.6 \mathrm{~W})$, and $\pm 5.4 \%( \pm 0.038)$, respectively.

To measure the internal flow of the hydraulic turbine using a PIV system, a solid-state laser (PIV Laser G6000, output $6 \mathrm{~W}$, and wavelength $532 \mathrm{~nm}$ ) with continuous oscillation was used as a light source. The light of the laser-sheet illuminated the central and vertical sections of the runner from a position vertically below the runner. During this operation, the laser-sheet light was reflected $90^{\circ}$ by a mirror. The thickness of the laser-sheet was about $1 \mathrm{~mm}$. Nylon 12 with a diameter of around $55 \mu \mathrm{m}$ and a specific gravity of 1.02 was used as tracer particles. A high-speed camera (Vision Research Inc., PHANTOM Miro M110) was used to photograph images in chronological order at a photography speed of $10,000 \mathrm{fps}$. The resolution is $448 \times 360$ pixels. Based on these images, with reference to the two two-dimensional components of the internal flow of the hydraulic turbine, PIV analysis was conducted by the direct cross-correlation method using a PIV analysis software from Flow Expert (Katokoken Co., Ltd.; Ver. 1.2.9).

6.2. Numerical Analysis Methods and Conditions. Along with this experiment, the actual turbine was analyzed using numerical analysis. The computational model of the actual turbine is shown in Figure 15. The analysis code used ANSYS CFX15.0 used in Section 4 and conducted three-dimensional 


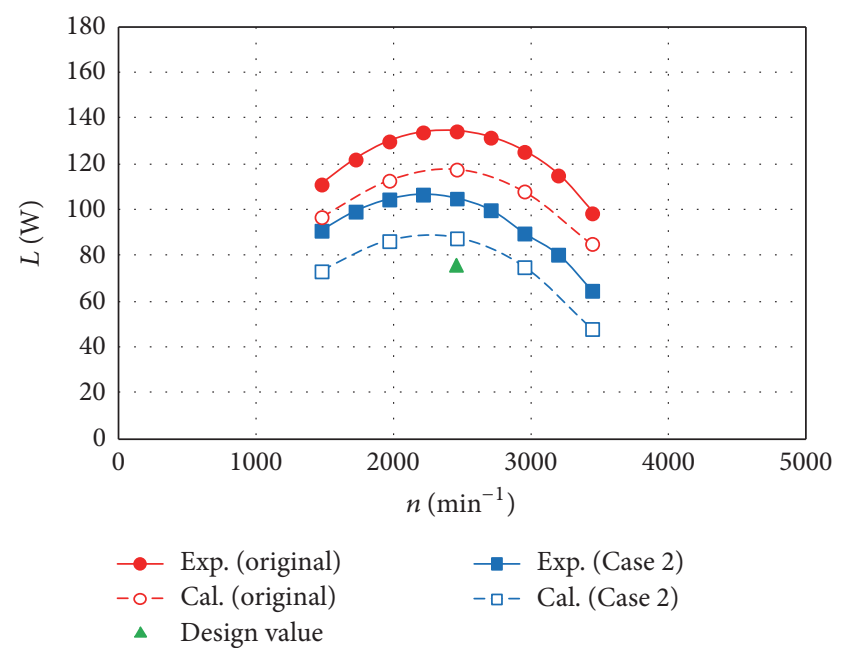

(a) Turbine output

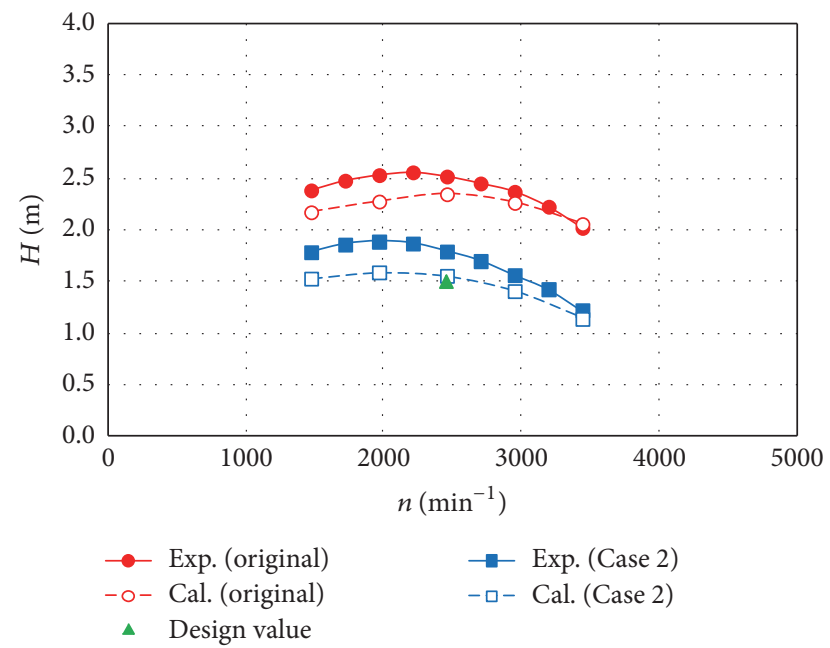

(b) Effective head

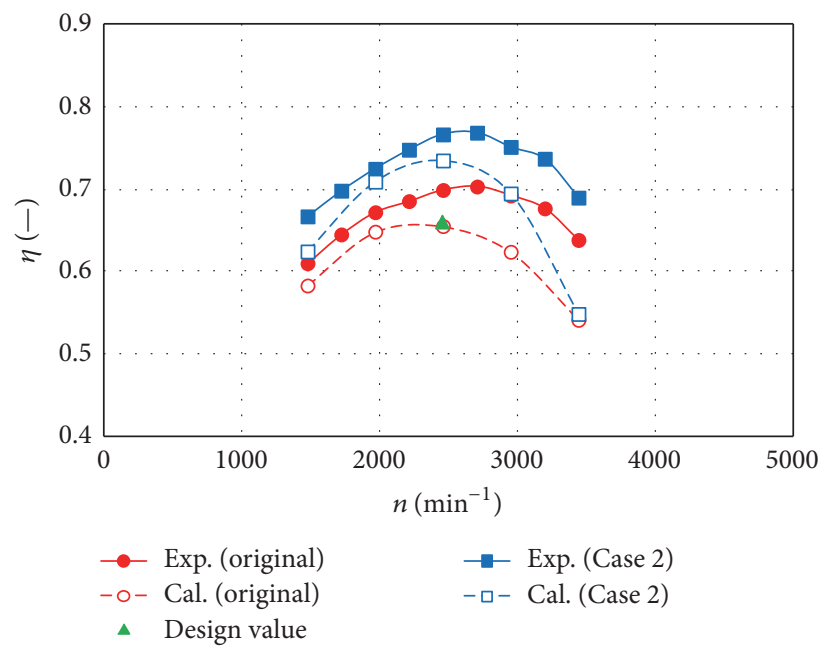

(c) Turbine efficiency

FIGURE 16: Turbine performances.

steady flow analyses. The governing equations, the turbulence model, the working fluid, the boundary conditions, and so forth are the same as those of Section 4. The computational grid of each runner was a tetrahedron, and the face size of the blade surface was $0.4 \mathrm{~mm}$. There were ten prism layers of the blade surface, and the size of the first layer was $0.007 \mathrm{~mm}$. The numbers of computational elements for original runner and Case 2 runner were approximately 2,530,000 and 3,150,000 elements, respectively. The computational grids of the guide vane used the same thing as Section 4. The total numbers of computational elements for original runner and Case 2 runner were approximately 5,810,000 and 6,430,000 elements, respectively.

6.3. Results and Discussion. The comparison of the performances of the original runner and Case 2 runner with regard to experimental values and calculated values is shown in Figures 16(a)-16(c). The turbine output, $L$, and the effective head,
$H$, from the experimental values of the original runner and Case 2 runner are all slightly larger than the calculated values. With regard to the turbine efficiency, $\eta$, the experimental and calculated values show qualitative agreement although there is a slight difference in the high rotational speed region. It is apparent that the turbine efficiency of Case 2 runner is significantly improved compared to that of the original runner and that Case 2 runner achieves an experimental value of $\eta=0.768$ for turbine efficiency, which is better than the target value.

With regard to the original runner and the Case 2 runner, the PIV measurement results of the absolute velocity vector in the central and vertical sections of the runners are illustrated in Figures 17(a) and 17(b), with the respective calculation results in Figures 18(a) and 18(b). Here, the rotational speed is $n=2460$. In addition, the PIV measurement results are obtained using time-averaged processing on the basis of the 12,196 images photographed (for approximately 50 


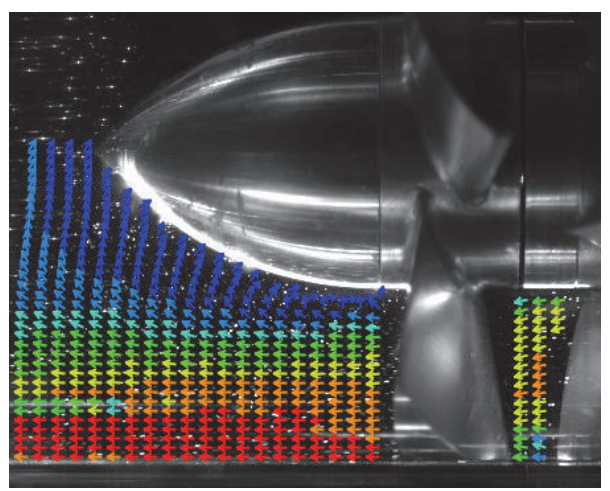

(a) Original

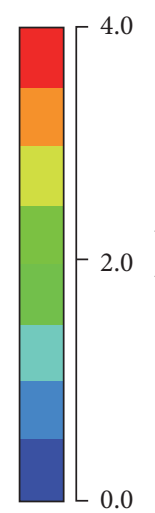

0.0

(1)

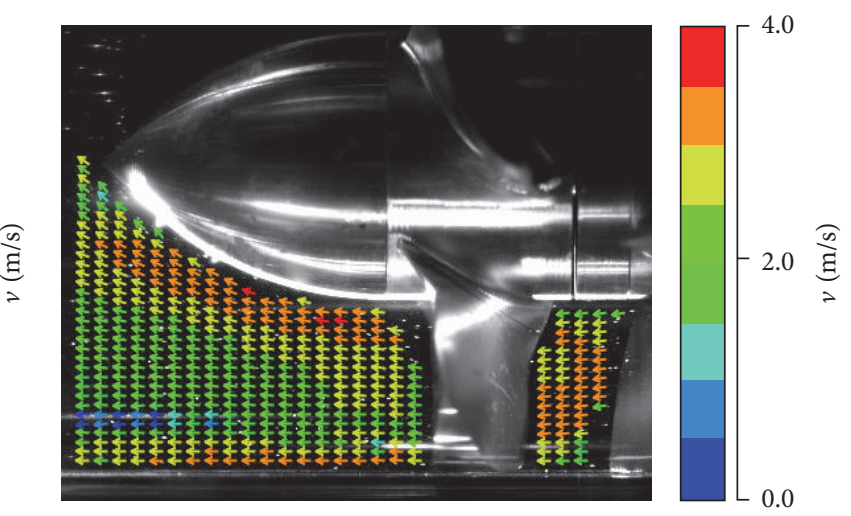

(b) Case 2

FIGURE 17: Absolute velocity vector $\left(n=2460 \mathrm{~min}^{-1}\right.$, Exp. $)$.

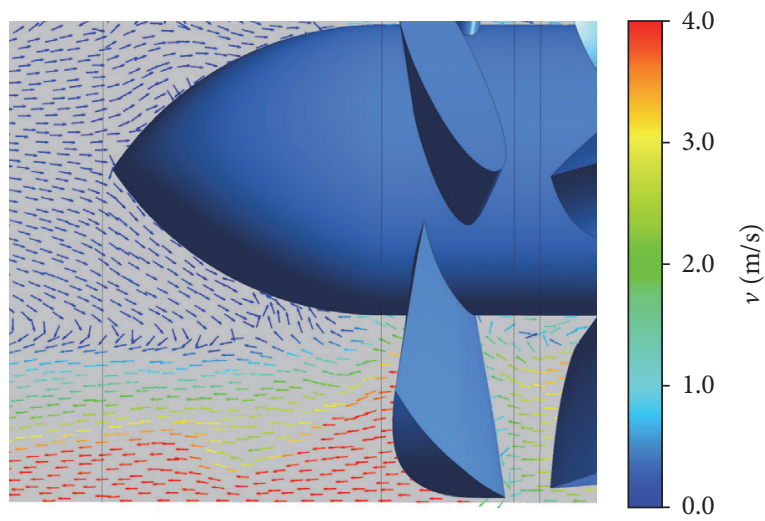

(a) Original

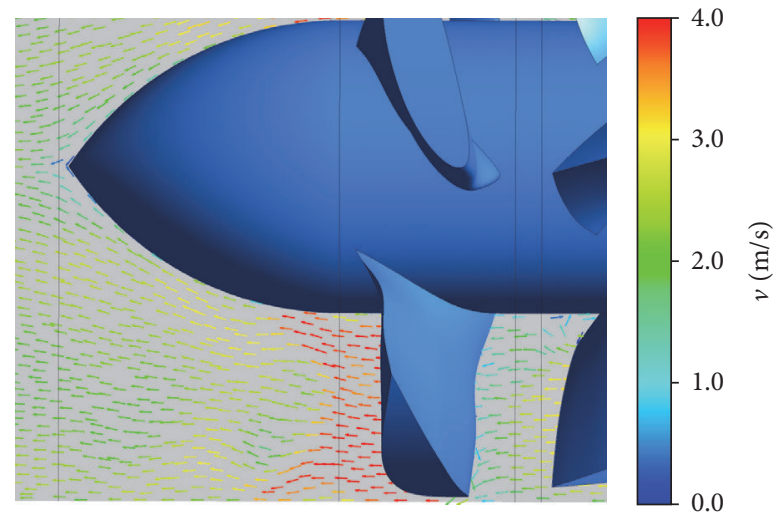

(b) Case 2

FIGURE 18: Absolute velocity vector $\left(n=2460 \mathrm{~min}^{-1}\right.$, Cal. $)$.

rotations of the runner). The flow at the outlet of the original runner in both the PIV measurement results and the calculation results is rapid on the tip side and slow on the hub side, which indicates nonuniformity. In addition, large-scale reverse flow is generated on the hub side. In contrast, Case 2 runner has no reverse flow at the outlet and is thus relatively uniform. Our design method is now validated.

As described above, using this design method, an axial flow runner with relatively high efficiency can be easily designed by altering the form of the runner only two or three times after producing a runner in a null state. At that time, we decide the unique form of the runner using quantitative data of the velocity distributions at the runner inlet and outlet (Figures 9(a) and 10(a)). Therefore, this is a simple method with a smaller calculation load in comparison with the optimized design method that uses a design of experiments and an optimization algorithm. In addition, our method can yield reliable results in comparison with the method wherein designers empirically repeat the changes of shapes by examining the analytical results from threedimensional internal flows.

\section{Conclusions}

We proposed a simple design method for axial flow runners using a combination of the conventional one-dimensional design method and the design method of axial flow velocity uniformization, with the support of three-dimensional flow analysis. We applied our design method to the runners of an ultra-small axial flow hydraulic turbine. The performance and internal flow of the hydraulic runner were investigated using numerical analysis and with an experiment. The conclusions are the following:

(1) For the original runner designed using a conventional one-dimensional design method, turbine efficiency is almost equal to the design values at a design rotational speed, but turbine output and effective head are significantly larger than their design values.

(2) Cases 1-3 runners designed with our design method are significantly improved in turbine efficiency compared to the original runner. Specifically, in the experiment, Case 2 runner achieves a turbine efficiency of 0.768 , which surpasses the target value. 
(3) The reason that the turbine efficiency of Case 2 runner shows a significant improvement in comparison with that of the original runner is that the axial component of absolute velocity is relatively uniform at the runner outlet, and as a result, the negative rotational flow is improved. Thus, the validity of our design method has been verified.

\section{Nomenclature}

$C_{L}:$ Lift coefficient

$D$ : Runner diameter $\mathrm{m}$

g: Gravitational acceleration $\mathrm{m} / \mathrm{s}^{2}$

$H$ : Effective head $\mathrm{m}$

$l$ : $\quad$ Chord length $\mathrm{m}$

L: Turbine output $\mathrm{W}$

$n$ : Rotational speed $\min ^{-1}$

$n_{s}$ : Specific speed $\min ^{-1}, \mathrm{~kW}, \mathrm{~m}$

$P$ : $\quad$ Static pressure $\mathrm{Pa}$

Q: Flow rate $\mathrm{m}^{3} / \mathrm{s}$

$r$ : Runner radius $m$

$t$ : Pitch $\mathrm{m}$

T: Torque N·m

$u$ : Circumferential velocity $\mathrm{m} / \mathrm{s}$

$v$ : Absolute velocity $\mathrm{m} / \mathrm{s}$

$w$ : Relative velocity $\mathrm{m} / \mathrm{s}$

$z$ : Number of blades.

\section{Greek Letters}

$\delta$ : Attack angle ${ }^{\circ}$

$\eta$ : Turbine efficiency $=L / \rho g Q H$

$\theta$ : Blade angle ${ }^{\circ}$

$\varphi$ : Dimensionless radial point $=\left(r-r_{h}\right) /\left(r_{t}-r_{h}\right)$

$\nu$ : Hub ratio $=D_{h} / D_{t}$

$\varepsilon$ : Drag-lift ratio

$\rho$ : Fluid density $\mathrm{kg} / \mathrm{m}^{3}$.

\section{Subscripts/Superscripts}

1: Runner inlet

2: Runner outlet

3: Guide vane inlet

4: Guide vane outlet

a: Axial component

$d$ : Design value

$g$ : Guide vane

$h$ : Hub

$i$ : Turbine inlet

$n$ : Numerical analysis value

$o$ : Turbine outlet

$t$ : Tip

$u$ : Circumferential component

': After the axial flow velocity uniformization

: Mean value between the runner inlet and outlet.

\section{Competing Interests}

The authors declare that there is no conflict of interests regarding the publication of this paper.

\section{Acknowledgments}

The authors acknowledge that a part of the study has been subsidized by JST A-STEP High-risk Challenge Type (Revitalization Promotion Type) and express their gratitude here.

\section{References}

[1] C. S. Kaunda, C. Z. Kimambo, and T. K. Nielsen, "A technical discussion on microhydropower technology and its turbines," Renewable and Sustainable Energy Reviews, vol. 35, pp. 445-459, 2014.

[2] A. Furukawa, S. Watanabe, D. Matsushita, and K. Okuma, "Development of ducted Darrieus turbine for low head hydropower utilization," Current Applied Physics, vol. 10, no. 2, pp. S128-S132, 2010.

[3] T. Ikeda, S. Iio, and K. Tatsuno, "Performance of nano-hydraulic turbine utilizing waterfalls," Renewable Energy, vol. 35, no. 1, pp. 293-300, 2010.

[4] A. Muis and P. Sutikno, "Design and simulation of very low head axial hydraulic turbine with variation of swirl velocity criterion," International Journal of Fluid Machinery and Systems, vol. 7, no. 2, pp. 68-79, 2014.

[5] H. M. Ramos, M. Simão, and K. N. Kenov, "Low-head energy conversion: a conceptual design and laboratory investigation of a microtubular hydro propeller," ISRN Mechanical Engineering, vol. 2012, Article ID 846206, 10 pages, 2012.

[6] R. Sonohata, J. Fukutomi, and T. Shigemitsu, "Study on contrarotating small-sized axial flow hydro turbine," Open Journal of Fluid Dynamics, vol. 2, pp. 318-323, 2012.

[7] T. Shigemitsu, Y. Takesihma, and J. Fukutomi, "Influence of spoke geometry on performance and internal flow of contrarotating small-sized hydroturbine," Turbomachinery, vol. 44, no. 2, pp. 89-97, 2016.

[8] E. Chica, S. Agudelo, and N. Sierra, "Lost wax casting process of the runner of a propeller turbine for small hydroelectric power plants," Renewable Energy, vol. 60, pp. 739-745, 2013.

[9] T. Nakamura, K. Sugishita, N. Ohtake, and N. Takasu, "Improvement of cavitation performance of francis turbine runner," Turbomachinery, vol. 33, no. 2, pp. 85-90, 2005.

[10] Y. Enomoto, S. Kurosawa, and T. Suzuki, “Design optimization of a francis turbine runner using multi-objective genetic algorithm," Turbomachinery, vol. 33, no. 12, pp. 732-737, 2005.

[11] Y. Enomoto, T. Nakamura, and S. Kurosawa, "Design optimization for francis turbine runners," Turbomachinery, vol. 41, no. 9, pp. 570-576, 2013.

[12] S. Tsuji, "Example exercises fluid machinery," Jitsugyotosyo, pp. 189-190, 1970.

[13] The Japan Society of Mechanical Engineers, Mechanical Engineers' Handbook Applications $\gamma 2$ Fluid Machinery, Maruzen, Tokyo, Japan, 2007.

[14] H. Matsumiya, T. Kogaki, M. Iida, and K. Kieda, "Development of a high performance airfoil," Turbomachinery, vol. 29, no. 9, pp. 519-524, 2001. 
[15] K. Imaichi, Y. Murakami, and H. Tsurusaki, The Foundation of the Pump Design with a Personal Computer, Japan Industrial Publishing, 1989.

[16] ANSYS Inc, ANSYS CFX-Solver Theoretical Guide, 2010.

[17] ANSYS, ANSYS CFX-Solver Modeling Guide, 2010. 


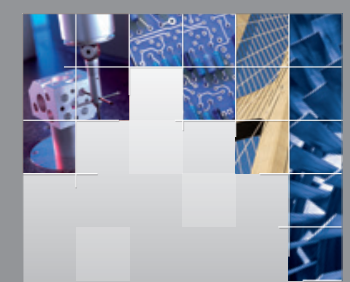

\section{Enfincering}
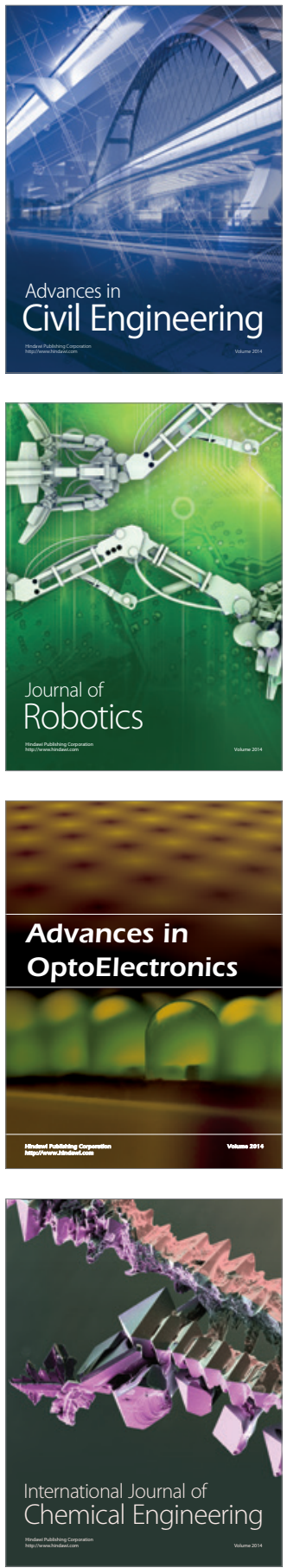

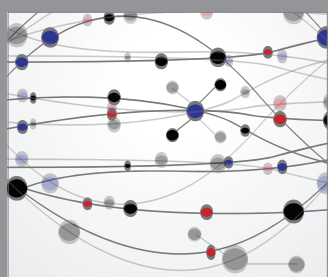

The Scientific World Journal

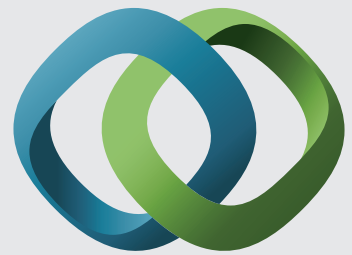

\section{Hindawi}

Submit your manuscripts at

http://www.hindawi.com
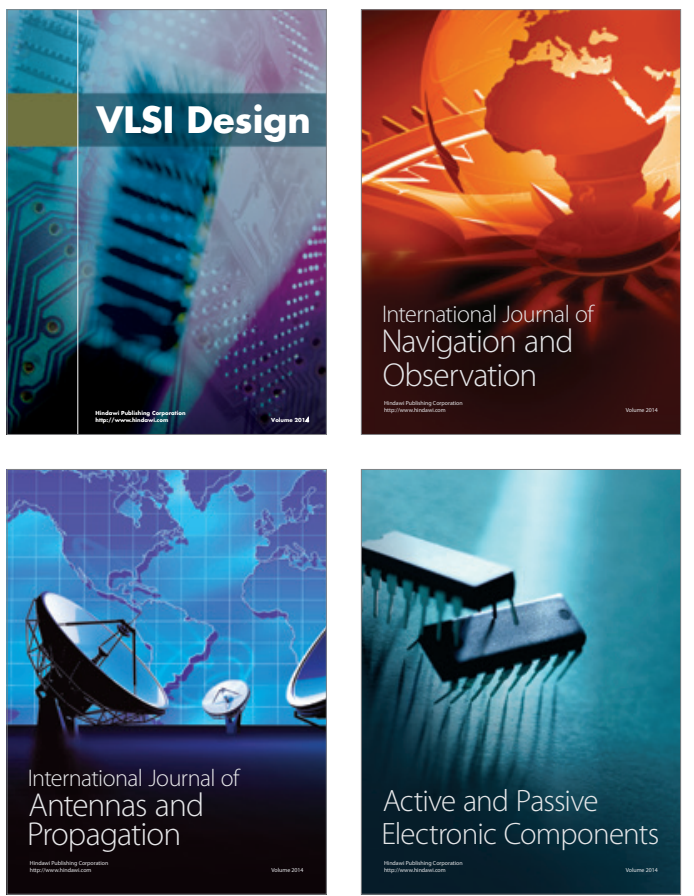
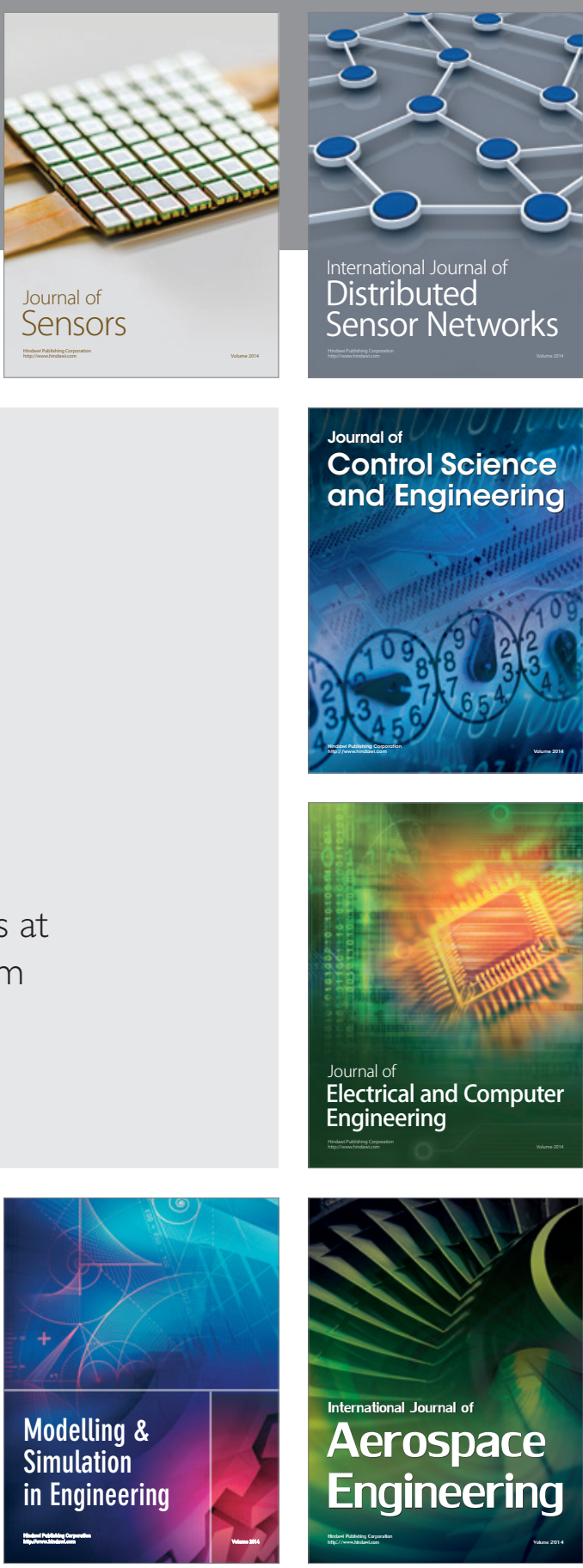

International Journal of

Distributed

Sensor Networks

Journal of

Control Science

and Engineering
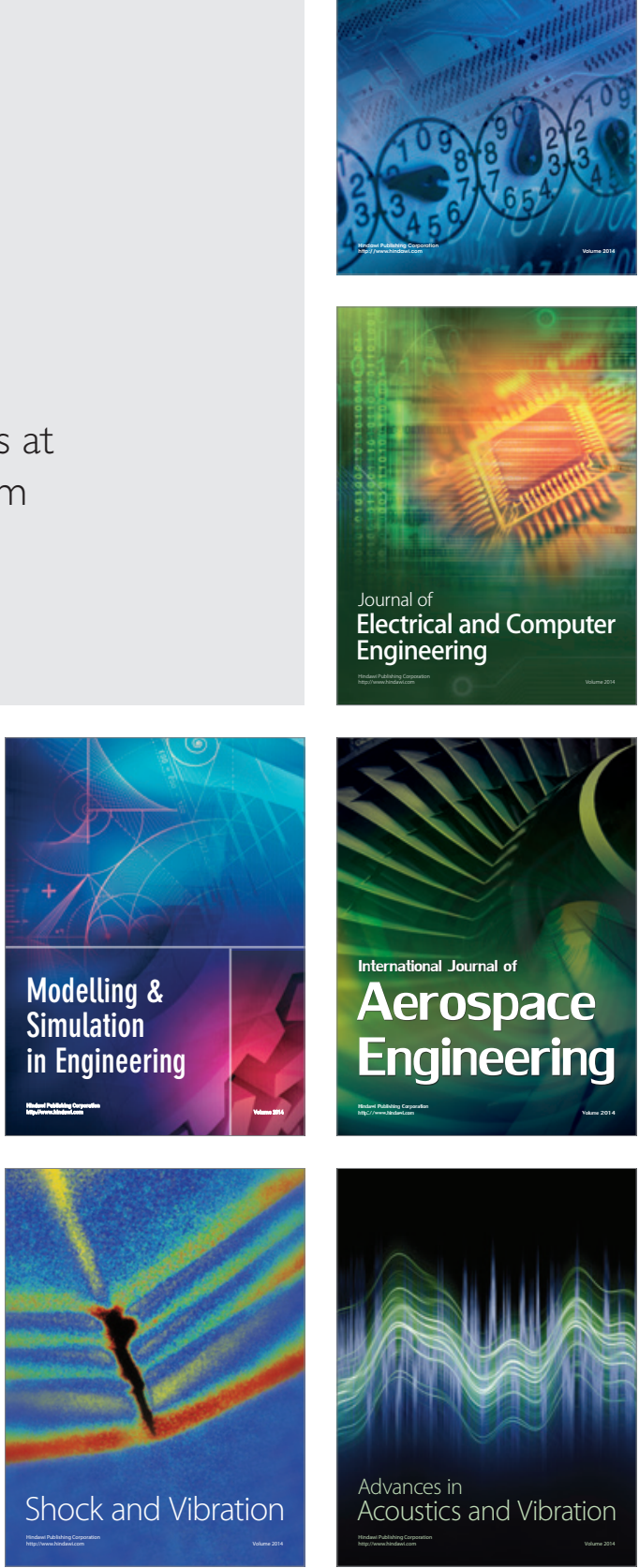